\title{
Viscoelastic substitute models for seismic attenuation caused by squirt flow and fracture leak off
}

\author{
Ralf Jänicke ${ }^{1}$, Beatriz Quintal ${ }^{2}$, Fredrik Larsson ${ }^{1}$, and Kenneth Runesson ${ }^{1}$
}

\begin{abstract}
We have investigated viscoelastic substitute models for seismic attenuation caused by fluid pressure diffusion in fluid-saturated porous media. Fluid pressure diffusion may locally occur associated with fracture leak off and/or squirt flow. We use a homogenization scheme with numerical model reduction (NMR), recently established in the literature, and we derive the corresponding viscoelastic material properties that are apparent at a larger scale (i.e., the observer scale). Moreover, we find that the rheology of the resulting viscoelastic model is of the Maxwell-Zener type. Based on a series of numerical experiments, we find that this method is able to accurately and efficiently predict the overall attenuation and stiffness moduli dispersion for a range of scenarios without resolving the substructure problem explicitly. Computational homogenization, together with NMR, can be useful to simulate seismic wave propagation using a viscoelastic substitute model that accurately reproduces the energy dissipation and dispersion of a heterogeneous medium in which squirt flow and/or fracture leak-off occurs.
\end{abstract}

\section{INTRODUCTION}

Computational technology has become an essential research and interpretation tool in geophysics. Seismic modeling is frequently used to characterize the seismic response of geologic formations. A frequently neglected but very important aspect of the seismic response from a fluid-saturated rock formation is the significant intrinsic attenuation and velocity dispersion in certain types of rocks (Carcione et al., 2010). This response is usually caused by waveinduced fluid flow at the mesoscopic and microscopic scales
(Müller et al., 2010). At the mesoscopic scale, wave-induced fluid flow is associated mainly with the presence of fractures or partial fluid saturation, whereas, at the microscopic scale, wave-induced fluid flow occurs as so-called squirt flow associated with the pore structure.

In a microscopic scenario, attenuation caused by squirt flow in the pores of a rock can be numerically modeled using the coupled equations for compressible fluid flow in the pores and elastic deformation in the embedding solid (Zhang and Toksöz, 2012; Quintal et al., 2016). Squirt flow occurs when compliant pores, connected to stiff or other compliant pores, are strongly deformed by the passing wave. Compliant pores have high aspect ratios and suffer maximal deformation and volume change when they are favorably oriented perpendicular to the wave-propagation direction. The strong deformation of a compliant pore induces a larger change in fluid pressure within this pore than that in the connected stiff pore or in the less favorably oriented compliant pore. The resulting fluid pressure gradient drives the dissipative squirt flow from one pore into the other one (O'Connell and Budiansky, 1977; Murphy et al., 1986; Gurevich et al., 2010).

In a mesoscopic scenario, similar phenomena can be observed in interconnected fractures. A squirt-type exchange of fluid occurs if one fracture is favorably oriented (e.g., nearly perpendicular to the P-wave wave propagation) and another fracture is less favorably oriented. In this case, seismic attenuation is associated with pressure diffusion due to redistribution of pore fluid that equilibrates the strong pressure gradients in the interconnected fractures (Rubino et al., 2013; Quintal et al., 2014; Vinci et al., 2014). Moreover, a second attenuation mechanism comes into play, often referred to as leak off. It is driven by fluid pressure difference between a fracture and the much stiffer embedding background and results in dissipative pressure diffusion in the latter (White, 1975; Brajanovski et al., 2005).

Attenuation in fractured rock can be modeled at the mesoscopic scale with Biot's $(1941,1962)$ equations of poroelasticity, which implies that the fractures are treated as a highly compressible, porous,

Manuscript received by the Editor 27 August 2018; revised manuscript received 25 April 2019; published ahead of production 09 May 2019; published online 20 June 2019.

${ }^{1}$ Chalmers University of Technology, Division for Material and Computational Mechanics, Gothenburg, Sweden. E-mail: ralf.janicke@chalmers.se (corresponding author); fredrik.larsson@ chalmers.se; kenneth.runesson@chalmers.se.

${ }^{2}$ University of Lausanne, Institute of Earth Sciences, Lausanne, Switzerland. E-mail: beatriz.quintal@unil.ch.

(C) 2019 Society of Exploration Geophysicists and American Association of Petroleum Geologists. All rights reserved. 
and permeable poroelastic medium (Brajanovski et al., 2005; Gurevich et al., 2009; Quintal et al., 2011; Rubino et al., 2013). Attenuation in fractured rock can also be modeled using coupled equations for compressible fluid flow in the fractures and poroelasticity in the embedding solid (Vinci et al., 2014).

The numerical upscaling procedures for microscopic and mesoscopic scenarios mentioned above are based on the assumption that the heterogeneous model behaves at a larger spatial scale as a homogeneous and apparently viscoelastic medium (Jänicke et al., 2015). The hydromechanical model is analyzed on the basis of its representative volume element (RVE). The diffusion lengths associated with the microscopic and mesoscopic processes are of the order of magnitude of the size of the corresponding heterogeneity and are usually much smaller than the wavelength of mechanical waves at seismic frequencies.

The described microscopic and mesoscopic dissipation scenarios are frequently encountered in subsurface geologic formations, as suggested by a series of laboratory-based studies (Adelinet et al., 2010; Tillotson et al., 2014; Pimienta et al., 2015; Subramaniyan et al., 2015). The numerical upscaling procedures mentioned above are useful to obtain the viscoelastic behavior of an RVE of a geologic material. However, to understand well the seismic response recorded on the surface, forward seismic wave wave-propagation modeling, or seismic modeling is an appropriate tool able to predict the seismic response for a certain assumed model describing the spatial distribution and properties of different formations. In seismic interpretation, iterative studies are conducted based on a range of assumed models. Resolving the microscopic pores or even the mesoscopic fractures in a macroscopic numerical model, having a much larger spatial scale than the RVE, would, however, be computationally extremely expensive, if feasible at all.

A reasonable solution is to substitute the heterogeneous hydromechanical medium with a homogeneous viscoelastic medium exhibiting an identical response in terms of attenuation and stiffness modulus dispersion. The challenge in this case is to find a method that allows for the identification of the viscoelastic substitute model in a numerically efficient way. The first step toward this goal has been accomplished with numerical upscaling procedures based on computational homogenization (Jänicke et al., 2015). This allows for obtaining the viscoelastic material response in a heuristic fashion using a volume averaging technique. Based on this framework, Jänicke et al. (2016) establish a numerical model reduction (NMR) procedure that can be used to derive viscoelastic substitute models for poroelastic media in a systematic and numerically highly efficient manner. This computational framework was recently extended to poroelastic media with embedded fluid-saturated fractures (Jänicke et al., 2019).

In the present contribution, our aim is, first, to use this novel NMR procedure proposed and described by Jänicke et al. (2019) to derive viscoelastic substitute models for a range of scenarios considering fluid pressure diffusion associated with the mesoscopic fracture leak-off and the mesoscopic or microscopic squirt flow. Second, we validate the results against reference computations and show that the proposed procedure is able to predict the material properties of the viscoelastic substitute model with high accuracy. Thus, the method represents, for example, a suitable way to execute forward simulation of seismic wave propagation with full access to pressure diffusion processes and with low computational efforts.

This paper is organized as follows: We supply the general ideas of how to identify viscoelastic substitute models for fluid pressure diffusion associated with fracture leak off and/or squirt flow without going into all technical details given in the precedent publications. We then investigate viscoelastic substitute models for seismic attenuation considering pressure diffusion in a range of $3 \mathrm{D}$ scenarios with interconnected mesoscopic fractures or microcracks embedded in a nonporous elastic background or with interconnected mesoscopic fractures embedded in a poroelastic background. Validation of the results is achieved by computational homogenization with NMR against reference computations with full resolution of the poroelastic RVEs with fractures. Finally, we summarize the results.

\section{IDENTIFICATION OF VISCOELASTIC SUBSTITUTE MODELS BY COMPUTATIONAL HOMOGENIZATION AND NMR}

In this section, we give a general overview of the computational homogenization and NMR concepts that are used for the simulations and discussions in this paper. Because it is our major interest to demonstrate the applicability and accuracy of the method to investigate seismic attenuation due to squirt flow and fracture leak off, we skip the extensive technical derivations and refer to Jänicke et al. (2019) for more information.

Attenuation of seismic waves is a phenomenon that occurs on multiple length scales. Our approach focuses on the length scales in which fractures or microcracks are present in the rock. Thus, we investigate mesoscopic and microscopic scales. Mesoscopic fractures and microcracks are much smaller than the length scale in which propagation of seismic waves occurs (the macroscopic scale). We investigate the mesoscopic- or microscopic-scale problem by defining volume elements that consist of fractures or microcracks embedded in a background material, which might be permeable or not. Because this volume element is considered to be representative for the entire structure, it is called RVE. The fundamental idea of computational homogenization is to establish a scale transition procedure that connects attenuation due to pressure diffusion within the RVE with viscoelastic attenuation of seismic waves on the macroscopic level (Jänicke et al., 2015). In other words, we propose a procedure that enables us to derive the homogeneous viscoelastic material properties of a macroscopic model that substitutes the heterogeneous RVE problem. What makes the procedure numerically efficient is that all the RVE computations are carried out once in advance as training computations. Once the model is trained, i.e., the viscoelastic properties are identified, the resulting viscoelastic material model can be used to simulate propagation and attenuation of seismic waves on the macrolevel without the need to resolve the RVE problem again.

To define the RVE problem, we use either linear elasticity or Biot's quasistatic poroelasticity equations of linear consolidation to describe the material properties of the embedding background rock. It is important to remark that, by this choice, we restrict our investigation to processes in the low-frequency limit, which is suitable for seismic attenuation. Hence, for the poroelastic background, Darcy's law is used to relate the seepage velocity of the saturating fluid to the fluid pressure gradient. Note that the case of linear elasticity is considered as a special case of poroelasticity when the material becomes impermeable.

The fractures are modeled as interfaces with zero thickness; however, we consider them to be mechanically and hydraulically open. The fracture aperture is accounted for as a material parameter 
(Vinci et al., 2014). The Poiseuille flow assumption allows for the modeling of seepage in the fractures via a Darcy-type constitutive relation. The hydromechanical coupling between the embedding background and fracture comprises momentum exchange as well as leak off of saturating fluid from the fractures into the background. Because we are interested in squirt-type processes driven by pore pressure diffusion at seismic frequencies, we further presume that the RVE is much smaller than the wavelength. Hence, we use undrained (here undrained refers to the fact that the amount of fluid inside the RVE remains constant although there might be periodic seepage across the boundary) periodic boundary conditions on the RVE. Altogether, this procedure enables us to apply a macroscopic strain or strain rate on the RVE, to solve a transient initial boundary value problem, and to extract the time-dependent stress response of the RVE by volume averaging.

In a second step, we make use of the problem's linearity and apply the superposition principle. More explicitly, we approximate the fluid pressure field $p(\mathbf{x}, t)$ in the RVE as a linear combination of a small number of $N$ pressure basis modes $p_{a}, a=1,2, \ldots, N$, such that

$$
p(\mathbf{x}, t)=\sum_{a=1}^{N} \bar{\chi}_{a}(t) p_{a}(\mathbf{x}),
$$

where the scalar parameter $N$ defines the dimension of the reduced basis and $\bar{\chi}_{a}(t)$ is called mode activity parameters whose temporal evolution needs to be evaluated. The set $p_{a}(\mathbf{x}), a=1,2, \ldots, N$, is called a reduced basis of the true pressure field $p(\mathbf{x}, t)$. Inserting this approximation into the combined continuity equation for fluid pressure diffusion in the fractures and the embedding background allows us to derive the evolution equations that control the activity $\bar{\chi}_{a}(t)$ of the pressure basis modes. As proved by Jänicke et al. (2019), the advantage of this method lies in the outcome that the resulting substitute model is of viscoelastic nature and, moreover, that the obtained set of evolution equations corresponds to a Maxwell-Zener rheology model. Thus, the resulting equation system defining the viscoelastic substitute model can be written as

$$
\begin{gathered}
\overline{\boldsymbol{\sigma}} \cdot \nabla=\mathbf{0}, \quad \overline{\boldsymbol{\sigma}}=\overline{\mathbf{C}}^{\mathrm{eff}}: \overline{\boldsymbol{\varepsilon}}+\sum_{a=1}^{N} \bar{\chi}_{a} \overline{\boldsymbol{\sigma}}_{a}, \\
\dot{\bar{\chi}}_{a}+\overline{\mathcal{C}}_{a} \bar{\chi}_{a}=\overline{\mathcal{D}}_{a, i j} \dot{\bar{\varepsilon}}_{i j}, \bar{\chi}_{a}(t=0)=0, a=1,2, \ldots, N .
\end{gathered}
$$

Here, we introduce the macroscopic stress and stiffness tensors $\overline{\boldsymbol{\sigma}}$ and $\overline{\mathbf{C}}^{\text {eff }}$ as well as the stresses $\overline{\boldsymbol{\sigma}}_{a}$ associated with the pressure modes $p_{a}$. The parameter $\overline{\mathcal{C}}_{a}$ represents the characteristic frequencies of the involved Maxwell chains. The parameter $\overline{\mathcal{D}}_{a, i j}$ defines the sensitivity of the particular Maxwell chain $a$ for the components $\dot{\bar{\varepsilon}}_{i j}$ of the macroscopic strain rate. The dimension $N$ of the set of reduced basis modes defines the number of Maxwell chains involved in the model and, thereby, to the dimension of the vectorvalued evolution equation 3 . It is important to remark that the constitutive quantities $\overline{\mathcal{C}}_{a}, \overline{\mathcal{D}}_{a, i j}, \overline{\mathbf{C}}^{\text {eff }}$, and $\overline{\boldsymbol{\sigma}}_{a}$ result from training computations on the RVE level as described by Jänicke et al. (2019). Once the viscoelastic model is identified, it can be used to execute, for example, forward simulations of seismic wave-propagation experiments without the need to go back to the RVE level.

\section{NUMERICAL EXPERIMENTS}

In this section, we validate and discuss the method for the identification of viscoelastic substitute models quantitatively. To this end, we consider a pair of interconnected circular fractures, one horizontal and the other one vertical, as illustrated in Figure 1. The fractures are filled with a compressible fluid. We first consider these fractures to be embedded in a nonporous and impermeable linear-elastic solid background, and thus pressure diffusion occurs only within the fractures. This scenario illustrates the case of interconnected mesoscopic fractures and interconnected microcracks. In a second example, we consider mesoscopic fractures to be embedded in a poroelastic background that is saturated with the same compressible fluid as the fracture; pressure diffusion occurs within the fractures as well as in the porous background.

We validate the attenuation behavior of the viscoelastic substitute models, identified according to our computational homogenization and NMR scheme, against reference computations. Reference computations are carried out with the finite-element method as stress relaxation experiments in the time domain on the basis of the fully resolved linear (poro)elastic RVEs with fractures (Quintal et al., 2011; Vinci et al., 2014). Hence, the respective strain components $\bar{\varepsilon}_{i j}$ are increased in a short-time interval and kept constant after reaching the plateau value. The results are transformed into the frequency domain via fast Fourier transform (FFT) and used to compute the frequency-dependent tangent stiffness operator:

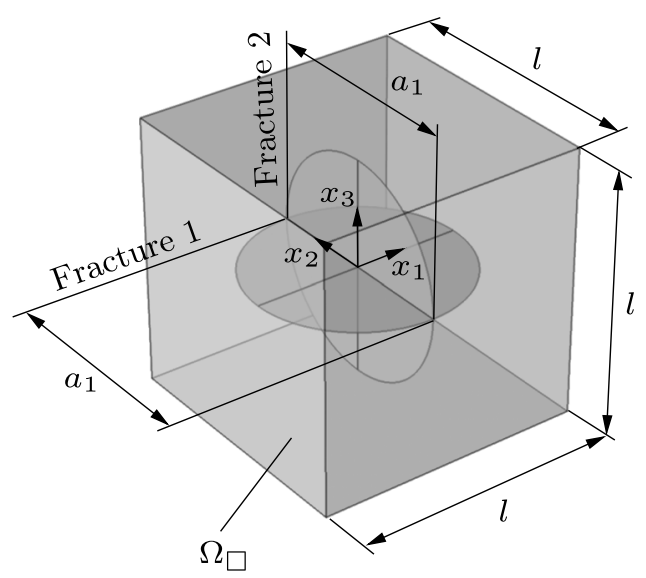

Figure 1. Cubic RVE in the domain $\Omega_{\square}$ with side length $l$ containing two perpendicular fractures with length $a_{1}$ and aperture $\tau_{0}$ in the $x_{1}-x_{2}$-plane (fracture 1$)$ and $x_{2}-x_{3}$-plane (fracture 2 ).

Table 1. Example 1: Material parameters of the RVE problem with impermeable embedding background.

Rock Fractures

\begin{tabular}{lcccc}
\hline Shear modulus background rock & $G$ & $(\mathrm{GPa})$ & 44 & - \\
Bulk modulus background rock & $K$ & $(\mathrm{GPa})$ & 40 & - \\
Bulk modulus saturating fluid & $K^{f}$ & $(\mathrm{GPa})$ & - & 2.4 \\
Effective dynamic viscosity & $\eta^{f R}$ & $(\mathrm{mPas})$ & - & 1 \\
Ratio RVE over fracture length & $l / a_{1}$ & - & 1.25 & - \\
\hline
\end{tabular}




$$
\bar{C}_{i j k l}(\omega)=\frac{\mathrm{d} \bar{\sigma}_{i j}(\omega)}{\mathrm{d} \bar{\varepsilon}_{k l}(\omega)} .
$$

In the case of the viscoelastic substitute model, the macroscopic stress components $\bar{\sigma}_{i j}$ are computed according to equation 2 . For the refer-
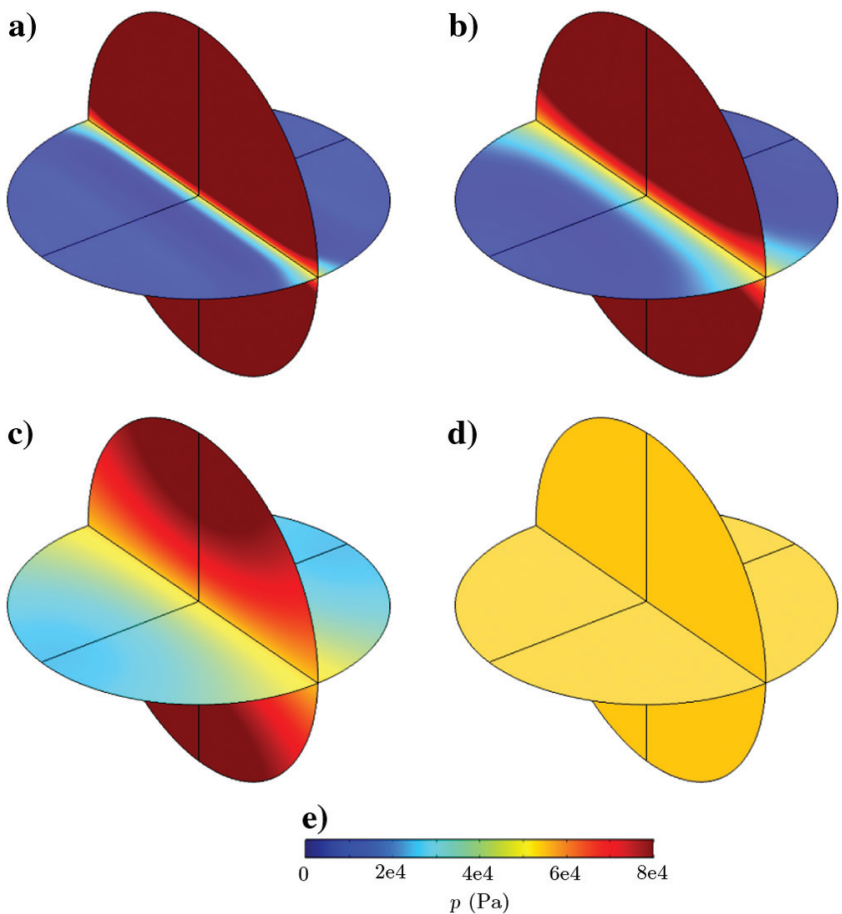

Figure 2. Example 1a: Snapshots of the evolving pressure field during a stress relaxation experiment with the loading $\bar{\varepsilon}_{11}(t)$.
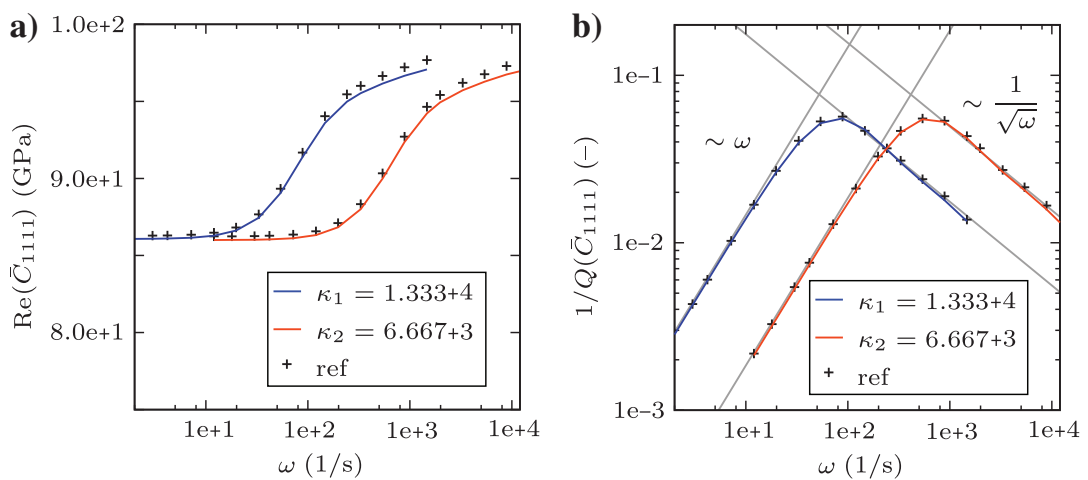

Figure 3. Example 1a: Constant aperture $\tau_{0}=\tau_{0, \max }$ but different aspect ratios: (a) real part and (b) inverse quality factor of the P-wave coefficient $\bar{C}_{1111}(\omega)$ using six internal variables to compute the resulting generalized Maxwell model. The overall transition frequencies are $\omega_{c}^{\kappa_{1}}=8.1 \mathrm{e}+11 / \mathrm{s}$ and $\omega_{c}^{\kappa_{2}}=6.5 \mathrm{e}+21 / \mathrm{s}$.

Table 2. Example 1a: Characteristic frequencies for models with aspect ratios $\kappa_{1}$ and $\kappa_{2}$. The branch $a=1$ represents the stationary case with $\omega_{c}=0$.

\begin{tabular}{lcccccc}
$\overline{\mathcal{C}}_{a}(1 / \mathrm{s})$ & $a=1$ & $a=2$ & $a=3$ & $a=4$ & $a=5$ & $a=6$ \\
\hline$\kappa_{1}=1.333 \mathrm{e}+4$ & 0 & $6.89 \mathrm{e}+3$ & $2.65 \mathrm{e}+3$ & $1.06 \mathrm{e}+3$ & $7.30 \mathrm{e}+2$ & $8.14 \mathrm{e}+1$ \\
$\kappa_{2}=6.667 \mathrm{e}+3$ & 0 & $5.43 \mathrm{e}+4$ & $2.09 \mathrm{e}+4$ & $8.36 \mathrm{e}+3$ & $5.79 \mathrm{e}+3$ & $6.48 \mathrm{e}+2$ \\
\hline
\end{tabular}

ence computations of the RVE problem, the stress components $\bar{\sigma}_{i j}$ are computed as volume average of the RVE stresses due to the imposed macroscopic strain $\bar{\varepsilon}_{k l}$.

\section{Numerical experiment 1: Impermeable rock matrix}

In the first numerical experiment, we consider the fluid-filled interconnected fractures to be embedded in a linear-elastic solid background. This is classically a microscopic scenario, but the corresponding physical pressure diffusion phenomenon is independent of the absolute spatial dimensions and controlled by the aspect ratio of the fractures (O'Connell and Budiansky, 1977). Thus, these fractures can represent mesoscopic fractures and microcracks. Here, we define the aspect ratio $\tau_{0}$ and the fracture length $a_{1}$ in relation to the side length $l$ of the cubic RVE. The material properties and the $l / a_{1}$ ratio are given in Table 1 . In the two subcases given below, we will consider, first, a scenario in which the aperture of the interconnected fractures is constant over the fracture length $\left(\tau_{0} \neq \tau_{0}(\mathbf{x})\right)$ and, second, a scenario in which the aperture varies $\left(\tau_{0}=\tau_{0}(\mathbf{x})\right)$.

\section{Numerical experiment 1a: Constant aperture}

We first investigate the scaling of the viscoelastic attenuation considering two different values for the aspect ratio of the fracture. We choose the aspect ratios $\kappa_{1}=a_{1} / \tau_{0,1}=1.333 \mathrm{e}+4$ and $\kappa_{2}=a_{1} / \tau_{0,2}=6.6667 \mathrm{e}+3$, i.e., $\kappa_{2}=2 \kappa_{1}$. All other quantities are identically chosen and given in Table 1 . Note that the fracture aperture is constant over its length, i.e., $\tau_{0}=\tau_{0 \text {,max }}$.

The workflow to identify the viscoelastic substitute model is according to Jänicke et al. (2019),

Step 1) Run training computations to identify the reduced basis modes $p_{a}$ for $\kappa_{1}$. In this particular case, the reduced basis obtained for $\kappa_{1}$ can be used for $\kappa_{2}$. (Note that this behavior is related to the particular scaling laws in this scenario. In general, one can expect a reduced basis to be sensitive for a change in the material parameters of the RVE. This would impose the need to reidentify the basis.)

Step 2) Compute the system matrices for $\kappa_{1}$ and $\kappa_{2}$, respectively.

The evolution of the fluid pressure field during stress relaxation is illustrated in Figure 2 for the reference case in which the RVE problem is solved with full resolution of the structure. We observe that the vertical fracture is compressed by the given loading $\bar{\varepsilon}_{11}$, which results in a high pressure, whereas the horizontal fracture remains uncompressed. Hence, pressure diffusion occurs within the fractures until the fluid pressure gradient is equilibrated.

In Figure 3, we show the real part and the inverse quality factor of the $\mathrm{P}$-wave modulus $\bar{C}_{1111}(\omega)$ under the loading $\bar{\varepsilon}_{111}$ for the two mentioned aspect ratios after FFT. The viscoelastic substitute models match well the reference computations for both cases, and the predictive quality of the viscoelastic models is very high for $\kappa_{1}$ as well as for $\kappa_{2}$. 
We also observe in Figure 3 that the dispersion and attenuation curves are only shifted in frequency, but their amplitudes are the same. We find that the transition frequencies of the two generalized Maxwell-Zener models are scaled by a factor eight such that

$$
\omega_{c}^{\kappa_{2}}=8 \omega_{c}^{\kappa_{1}}
$$

Hence, a reusage of basis modes as for the two cases in experiment $1 \mathrm{a}$ is not possible.

Also in this case, we observe that the viscoelastic substitute model approximates the reference computation with high fidelity. Moreover, we find that the fracture aperture has an effect on the attenuation behavior. More explicitly, we can see that the transition
This is related to the ratio of aspect ratios, i.e., $\left(\kappa_{2} / \kappa_{1}\right)^{3}=2^{3}=8$. The same relation holds for all characteristic frequencies $\overline{\mathcal{C}}_{a}$ associated with the individual chains of the resulting generalized Maxwell model, with values given in Table 2.

Interestingly, the transition frequencies, i.e., the frequencies at which the attenuation is maxi$\mathrm{mal}, \omega_{c}^{\kappa_{1}}=8.1 \mathrm{e}+11 / \mathrm{s}$ and $\omega_{c}^{\kappa_{2}}=6.5 \mathrm{e}+21 / \mathrm{s}$ observed in Figure 3, correspond approximately to the lowest characteristic frequency $\overline{\mathcal{C}}_{6}$ of the viscoelastic substitute model (Table 2). Hence, the faster characteristic frequencies $\overline{\mathcal{C}}_{a}$, $a=2, \ldots, 5$, describe the transition toward the high-frequency limit. Moreover, we observe in the low-frequency limit that $1 / Q$ scales with $\omega$ and in the high-frequency limit with $1 / \sqrt{\omega}$, as predicted by squirt flow analytical solutions considering saturation with a liquid (Gurevich et al., 2010). If we, in contrast, investigate the simplest case of a Maxwell-Zener model consisting of one single Maxwell chain in parallel with an elastic spring, it is easy to show that the attenuation $1 / Q$ scales with $\omega$ in the low-frequency limit and with $1 / \omega$ in the high-frequency limit. Thus, additional Maxwell chains with larger characteristic frequencies are required to recover the correct slope toward higher frequencies for simple squirt flow models, such as the one illustrated in Figure 1, or to recover the broader frequency-dependent behavior of fracture networks having more complex spatial distributions.

\section{Numerical experiment 1b: Variable aperture}

In the second subcase, we define the fracture aperture variable along the fracture length as

$$
\tau_{0}^{v}=\tau_{0, \max }\left(1-\frac{2 r}{a_{1}}\right) .
$$

Hence, the fracture aperture scales linearly with the radius $r$ of the circular fractures between $\tau_{0}^{v}(r=0)=\tau_{0, \max }$ in the center and $\tau_{0}^{v}\left(r=a_{1} / 2\right)=0$ at the (circular) fracture tips. In this experiment, we choose $\tau_{0, \max }=\tau_{0,1}=a_{1} / \kappa_{1}$ with $\kappa_{1}=1.333 e+4$. All other material properties remain unchanged and correspond to Table 1 . In Figure 4, we show the real part and the inverse quality factor of the P-wave modulus $\bar{C}_{1111}$ for fractures having such variable apertures as well as the corresponding results for the constant apertures already shown as the blue curves in Figure 3.

It is important to remark that, in contrast to experiment $1 \mathrm{a}$, both choices for the aperture require an individual identification of the reduced basis and the corresponding viscoelastic substitute models.
Table 4. Example 2: Material parameters of the RVE problem with permeable background rock $\left(1 \mathrm{mD} \approx 1 \mathrm{e}-15 \mathrm{~m}^{2}\right)$.

Rock Fractures
Shear modulus rock matrix

Bulk modulus rock matrix

Bulk modulus solid grains

Intrinsic permeability rock matrix

Porosity rock matrix

Bulk modulus saturating fluid

Effective dynamic viscosity

RVE length

Fracture length

Fracture aperture

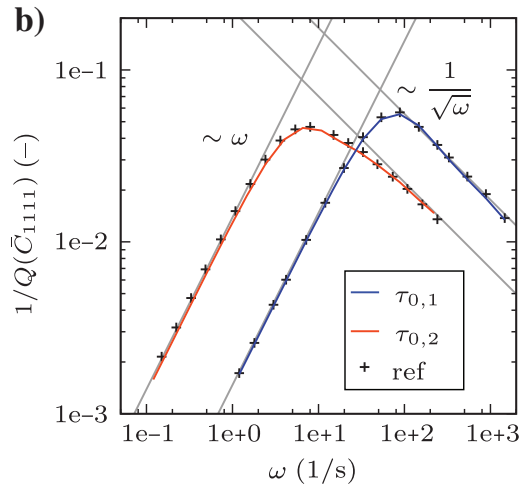

(b) inverse quality factor of the P-wave coefficient $\bar{C}_{1111}(\omega)$ for a constant aperture $\tau_{0,1}$ and the variable aperture 列

Table 3. Example 1b: Characteristic frequencies for models with different fracture apertures $\tau_{0,1}$ and $\tau_{0}^{v 1}$.

\begin{tabular}{lcccccc}
$\overline{\mathcal{C}}_{a}(1 / \mathrm{s})$ & $a=1$ & $a=2$ & $a=3$ & $a=4$ & $a=5$ & $a=6$ \\
\hline$\tau_{0,1}$ & 0 & $6.89 \mathrm{e}+3$ & $2.65 \mathrm{e}+3$ & $1.06 \mathrm{e}+3$ & $7.30 \mathrm{e}+2$ & $8.14 \mathrm{e}+1$ \\
$\tau_{0}^{v 1}$ & 0 & $1.82 \mathrm{e}+3$ & $7.69 \mathrm{e}+2$ & $3.48 \mathrm{e}+2$ & $1.58 \mathrm{e}+2$ & $8.83 \mathrm{e}+1$ \\
\hline & & & $a=7$ & $a=8$ & $a=9$ \\
\hline$\tau_{0,1}$ & & & - & - & - \\
$\tau_{0}^{v 1}$ & & & & $4.37 \mathrm{e}+1$ & $1.76 \mathrm{e}+1$ & $6.79 \mathrm{e}+0$ \\
\hline
\end{tabular}



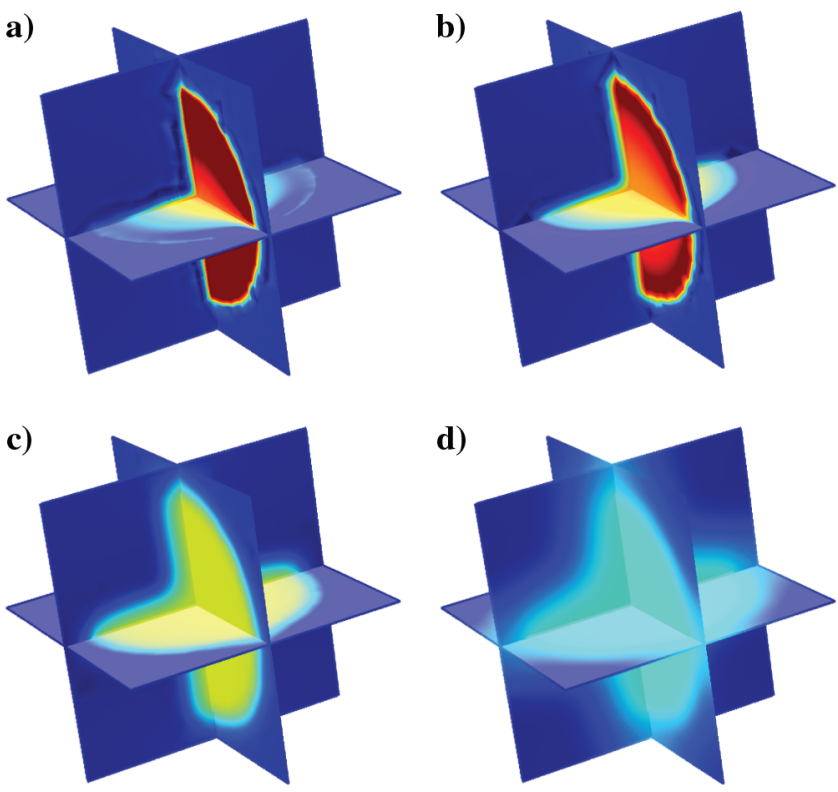

e)

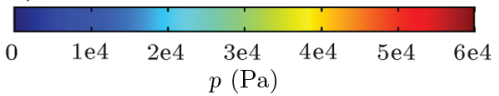

Figure 5. Example 2: Snapshots of the evolving pressure field during stress relaxation experiment under $\bar{\varepsilon}_{11}(t)$.

Table 5. Example 2: Characteristic frequencies for the fracture model with leak off.

$$
\begin{array}{rcccccc}
\overline{\mathcal{C}}_{a}(1 / \mathrm{s}) & a=1 & a=2 & a=3 & a=4 & a=5 & a=6 \\
\hline 0 & 3.71 \mathrm{e}+1 & 3.05 \mathrm{e}+1 & 1.16 \mathrm{e}+1 & 1.14 \mathrm{e}+1 & 4.96 \mathrm{e}-0 \\
a=7 & a=8 & a=9 & a=10 & a=11 & a=12 \\
3.77 \mathrm{e}-0 & 1.83 \mathrm{e}-0 & 1.59 \mathrm{e}-0 & 7.43 \mathrm{e}-1 & 6.52 \mathrm{e}-1 & 3.32 \mathrm{e}-1 \\
a=13 & a=14 & a=15 & a=16 & a=17 & a=18 \\
1.79 \mathrm{e}-1 & 1.77 \mathrm{e}-1 & 9.43 \mathrm{e}-2 & 5.19 \mathrm{e}-2 & 3.95 \mathrm{e}-2 & 1.87 \mathrm{e}-2
\end{array}
$$

frequency in the subcase with variable aperture is shifted by approximately one order of magnitude toward lower frequencies. This is reasonable because we have chosen $\tau_{0}^{v} \leq \tau_{0 \text {,max }}$, which reduces the effective permeability of the fractures. Furthermore, the pressure diffusion phenomenon for the case with variable fracture aperture requires a more complex substitute model (nine Maxwell chains instead of six) (see Table 3). This is a consequence of the wider attenuation peak observed for the variable aperture (see Figure 4b).

\section{Numerical experiment 2: Permeable rock matrix}

Although using the RVE shown in Figure 1, we now increase the complexity of the problem and include fluid pressure diffusion in the embedding background due to leak off of fluid from the fractures into the porous background. We use the variable aperture as defined in equation 6 . Note that, allowing for leak-off and pressure diffusion in the background rock, the diffusion length is constrained not only by the fracture length but also by the chosen length of the RVE sides.

To prove the power of the proposed identification method, we choose the material and geometric parameters given in Table 4 such that the two attenuation processes, i.e., squirt-type pressure diffusion within the fractures and pressure diffusion due to leak off in the porous background, occur at significantly different transition frequencies. The latter is strongly affected by (1) the permeability in the background material and (2) the overall size of the used RVE, i.e., the distance between parallel fractures in the periodic medium composed of an assembly of such a unit cell.

The evolution in time of the fluid pressure field observed in the fully resolved RVE under stress relaxation is depicted in Figure 5 for four exemplary chosen time steps. For the earlier times, we observe the onset of squirt-type fluid pressure diffusion in the fractures. At later times, fluid pressure diffusion in the embedding background becomes dominant and equilibrates the remaining pressure gradients. We identify the viscoelastic substitute model for this example and find a very good approximation of the reference computations if we use a reduced basis consisting of 18 pressure modes. Hence, the resulting viscoelastic model comprises 18 Maxwell chains. The characteristic frequencies are given in Table 5.

The resulting frequency-dependent attenuation and stiffness modulus dispersion of the viscoelastic substitute model are given in Figure 6. We observe a pronounced attenuation peak at approximately the same transition frequency as observed in the previous example (experiment $1 \mathrm{~b}$ ) with variable aperture and impermeable background. The reason is that this peak results from the (fast) squirt-type fluid pressure diffusion within the fracture, the same phenomenon as in the previous example. The frequency interval with almost constant attenuation $1 / Q$ between 0.05 and $11 / \mathrm{s}$, on the other hand, is dominated by fluid pressure diffusion in the embedding background. Here, we again observe that the viscoelastic substitute model approximates the fully resolved reference computation with high accuracy. Note that this high number of Maxwell chains is required for two reasons. First, the pressure diffusion patterns are highly complex (diffusion in fractures + leak off + diffusion in background rock), which is reflected by the fact that attenuation and dispersion occur in a
Figure 6. Example 2: (a) Real part and (b) inverse quality factor of the P-wave coefficient $\bar{C}_{1111}(\omega)$ using 18 internal variables.

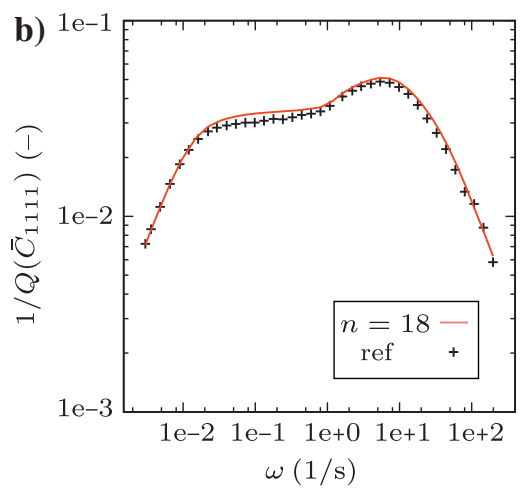


wide frequency range. Second, it is important to remark that, in particular, the background diffusion processes are strongly influenced by the distinct anisotropy of the chosen structure.

\section{CONCLUSION}

In this contribution, we used a computational scheme comprising homogenization and NMR that allows for the identification of viscoelastic substitute models for seismic attenuation in porous rock in a numerically efficient way. We identified viscoelastic substitute models for several scenarios. The apparently viscoelastic material properties are associated with fluid pressure diffusion due to squirt flow in interconnected mesoscopic fractures or microcracks as well as due to leak off from mesoscopic fractures into the embedding background. We showed that, in all numerical experiments, the computational identification technique provides highly accurate predictions of the seismic attenuation behavior of the underlying substructure. Furthermore, we found that the width of the attenuation peak in the frequency domain as well as the anisotropy of the chosen problems influence the required number of viscoelastic variables.

The main advantage of the presented identification strategy is that it enables us to execute forward simulations of seismic wave propagation in complex scenarios by using homogeneous viscoelastic substitute models to replace the heterogeneous poroelastic subdomains exhibiting pressure diffusion processes. The method is numerically highly efficient, with the identification procedure only requiring transient training computations on the RVE level. These computations are executed "offline"; i.e., they are executed once in advance. Once the reduced basis modes are identified, the "online" computations on the substructure do no longer burden the wave-propagation simulation.

\section{ACKNOWLEDGMENTS}

This project is funded by the Swedish Research Council (VR) under the grant no. 2017-05192. The financial support is gratefully acknowledged.

\section{DATA AND MATERIALS AVAILABILITY}

Data associated with this research are available and can be obtained by contacting the corresponding author.

\section{REFERENCES}

Adelinet, M., J. Fortin, Y. Guéguen, A. Schubnel, and L. Geoffroy, 2010, Frequency and fluid effects on elastic properties of basalt: Experimental investigations: Geophysical Research Letters, 37, L02303, doi: 10.1029/ 2009GL041660.

Biot, M., 1941, General theory of three-dimensional consolidation: Journal of Applied Physics, 12, 155-164, doi: 10.1063/1.1712886.

Biot, M., 1962, Mechanics of deformation and acoustic propagation in porous media: Journal of Applied Physics, 33, 1482-1498, doi: 10 $.1063 / 1.1728759$.
Brajanovski, M., B. Gurevich, and M. Schoenberg, 2005, A model for $\mathrm{P}$-wave attenuation and dispersion in a porous medium permeated by aligned fractures: Geophysical Journal International, 163, 372-384, doi: 10.1111/j.1365-246X.2005.02722.x.

Carcione, J., C. Morency, and J. E. Santos, 2010, Computational poroelasticity - A review: Geophysics, 75, no. 5, 75A229-75A243, doi: 10.1190/ 1.3474602 .

Gurevich, B., M. Brajanovski, R. Galvin, T. Muller, and J. Toms-Stewart, 2009, P-wave dispersion and attenuation in fractured and porous reservoirs - Poroelasticity approach: Geophysical Prospecting, 57, 225237, doi: 10.1111/j.1365-2478.2009.00785.x.

Gurevich, B., D. Makarynska, O. de Paula, and M. Pervukhina, 2010, A simple model for squirt-flow dispersion and attenuation in fluid-saturated granular rocks: Geophysics, 75, no. 6, N109-N120, doi: 10.1190/1 .3509782 .

Jänicke, R., F. Larsson, H. Steeb, and K. Runesson, 2016, Numerical identification of a viscoelastic substitute model for heterogeneous poroelastic media by a reduced order homogenization approach: Computer Methods in Applied Mechanics and Engineering, 298, 108-120, doi: 10.1016/j cma.2015.09.024.

Jänicke, R., B. Quintal, F. Larsson, and K. Runesson, 2019, Identification of viscoelastic properties from numerical model reduction of pressure diffusion in fluid-saturated porous rock with fractures: Computational Mechanics, 63, 49-67, doi: 10.1007/s00466-018-1584-7.

Jänicke, R., B. Quintal, and H. Steeb, 2015, Numerical homogenization of mesoscopic loss in poroelastic media: European Journal of Mechanics A/Solids, 49, 382-395, doi: 10.1016/j.euromechsol.2014.08.011.

Müller, T., B. Gurevich, and M. Lebedev, 2010, Seismic wave attenuation and dispersion resulting from wave-induced flow in porous rocks - $\mathrm{A}$ review: Geophysics, 75, no. 5, A147-A164, doi: 10.1190/1.3463417.

Murphy, W., K. Winkler, and R. Kleinberg, 1986, Acoustic relaxation in sedimentary rocks: Dependence on grain contacts and fluid saturation: Geophysics, 51, 757-766, doi: 10.1190/1.1442128.

O'Connell, R., and B. Budiansky, 1977, Viscoelastic properties of fluid-saturated cracked solids: Journal of Geophysical Research, 82, 5719-5735, doi: 10.1029/JB082i036p05719.

Pimienta, L., J. Fortin, and Y. Gueguen, 2015, Bulk modulus dispersion and attenuation in sandstones: Geophysics, 80, no. 2, D111-D127, doi: 10 $.1190 /$ geo2014-0335.1.

Quintal, B., R. Jänicke, J. Rubino, H. Steeb, and K. Holliger, 2014, Sensitivity of S-wave attenuation to the connectivity of fractures in fluid-saturated rocks: Geophysics, 79, no. 5, WB15-WB24, doi: 10.1190/geo20130409.1 .

Quintal, B., J. Rubino, E. Caspari, and K. Holliger, 2016, A simple hydromechanical approach for simulating squirt-type flow: Geophysics, 81, no. 4, D335-D344, doi: 10.1190/geo2015-0383.1.

Quintal, B., H. Steeb, M. Frehner, and S. Schmalholz, 2011, Quasi-static finite-element modeling of seismic attenuation and dispersion due to wave-induced fluid flow in poroelastic media: Journal of Geophysical Research, 116, B01201, doi: 10.1029/2010JB007475.

Rubino, J., L. Guarracino, T. Müller, and K. Holliger, 2013, Do seismic waves sense fracture connectivity?: Geophysical Research Letters, 40, 692-696, doi: 10.1002/grl.50127.

Subramaniyan, S., B. Quintal, C. Madonna, and E. H. Saenger, 2015, Laboratory-based seismic attenuation in Fontainebleau sandstone: Evidence of squirt flow: Journal of Geophysical Research, 120, 7526-7535.

Tillotson, P., M. Chapman, J. Sothcott, A. Best, and X.-Y. Li, 2014, Pore fluid viscosity effects on P- and S-wave anisotropy in synthetic silicacemented sandstone with aligned fractures: Geophysical Prospecting, 62, 1238-1252, doi: $10.1111 / 1365-2478.12194$.

Vinci, C., J. Renner, and H. Steeb, 2014, On attenuation of seismic waves associated with flow in fractures: Geophysical Research Letters, 41, 7515-7523, doi: 10.1002/2014GL061634.

White, J., 1975, Computed seismic speeds and attenuation in rocks with partial gas saturation: Geophysics, 40, 224-232, doi: 10.1190/1.1440520.

Zhang, Y., and M. Toksöz, 2012, Computation of dynamic seismic responses to viscous fluid of digitized three-dimensional Berea sandstones with a coupled finite-difference method: The Journal of the Acoustical Society of America, 132, 630-640, doi: 10.1121/1.4733545. 